\title{
Constraining the near-core rotation of the $\gamma$ Doradus star 43 Cygni using BRITE-Constellation data ${ }^{\star, \star \star}$
}

\author{
K. Zwintz ${ }^{1, \star \star \star}$, T. Van Reeth ${ }^{2,3}$, A. Tkachenko ${ }^{2}$, S. Gössl ${ }^{1}$, A. Pigulski ${ }^{4}$, R. Kuschnig ${ }^{5}$, G. Handler ${ }^{6}$, A. F. J. Moffat ${ }^{7}$, \\ A. Popowicz ${ }^{8}$, G. Wade ${ }^{9}$, and W. W. Weiss ${ }^{10}$
}

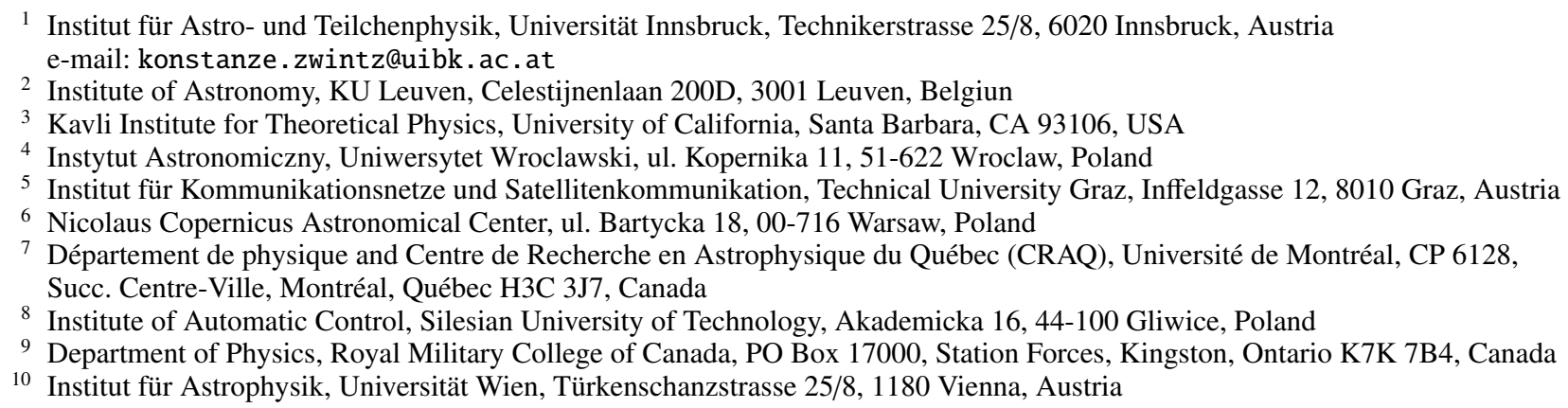

Received 16 August 2017 / Accepted 6 October 2017

\begin{abstract}
Context. Photometric time series of the $\gamma$ Doradus star 43 Cyg obtained with the BRITE-Constellation nano-satellites allow us to study its pulsational properties in detail and to constrain its interior structure.

Aims. We aim to find a $g$-mode period-spacing pattern that allows us to determine the near-core rotation rate of 43 Cyg and redetermine the star's fundamental atmospheric parameters and chemical composition.

Methods. We conducted a frequency analysis using the 156-day long data set obtained with the BRITE-Toronto satellite and employed a suite of MESA/GYRE models to derive the mode identification, asymptotic period-spacing, and near-core rotation rate. We also used high-resolution spectroscopic data with high signal-to-noise ratio obtained at the $1.2 \mathrm{~m}$ Mercator telescope with the HERMES spectrograph to redetermine the fundamental atmospheric parameters and chemical composition of 43 Cyg using the software Spectroscopy Made Easy (SME).

Results. We detected 43 intrinsic pulsation frequencies and identified 18 of them to be part of a period-spacing pattern consisting of prograde dipole modes with an asymptotic period-spacing $\Delta \Pi_{l=1}$ of $2970_{-570}^{+700} \mathrm{~s}$. The near-core rotation rate was determined to be $f_{\text {rot }}=0.56_{-0.14}^{+0.12} \mathrm{~d}^{-1}$. The atmosphere of 43 Cyg shows solar chemical composition at an effective temperature, $T_{\text {eff }}$, of $7150 \pm 150 \mathrm{~K}$, a $\log g$ of $4.2 \pm 0.6 \mathrm{dex}$, and a projected rotational velocity, $v \sin i$, of $44 \pm 4 \mathrm{~km} \mathrm{~s}^{-1}$.

Conclusions. The morphology of the observed period-spacing patterns shows indications of a significant chemical gradient in the stellar interior.
\end{abstract}

Key words. asteroseismology - stars: individual: 43 Cygni - stars: interiors - stars: oscillations - stars: fundamental parameters

\section{Introduction}

$\gamma$ Doradus stars are intermediate-mass stars, with typical masses between $1.4 M_{\odot}$ and $2.0 M_{\odot}$. They cover the transition region between low-mass stars possessing radiative cores and convective envelopes to high-mass stars, which have convective cores and radiative envelopes. $\gamma$ Doradus pulsators exhibit non-radial

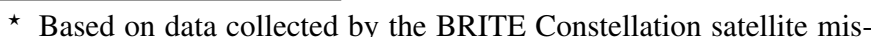
sion, designed, built, launched, operated and supported by the Austrian Research Promotion Agency (FFG), the University of Vienna, the Technical University of Graz, the Canadian Space Agency (CSA), the University of Toronto Institute for Aerospace Studies (UTIAS), the Foundation for Polish Science \& Technology (FNiTP MNiSW), and National Science Centre (NCN).

$\star \star$ The light curves (in tabular form) are only available at the CDS via anonymous ftp to cdsarc.u-strasbg. fr (130.79.128.5) or via http://cdsarc.u-strasbg.fr/viz-bin/qcat?J/A+A/608/A103 $\star \star \star$ FWF Elise Richter fellow.
}

gravity $(g)$ and/or gravito-inertial modes, with observed pulsation periods between 0.3 and 3 days (Kaye et al. 1999) that are excited by the convective flux-blocking mechanism at the bottom of the convective envelope (Guzik et al. 2000; Dupret et al. 2005). These pulsations are most sensitive to the characteristics of the deep stellar interior. The pulsation periods are predicted to be equidistantly spaced in the asymptotic regime $(n \gg l)$ for a non-rotating chemically homogeneous star with a convective core and a radiative envelope (Tassoul 1980). The presence of a chemical gradient at the edge of the convective core induces nonuniform variations in the spacing pattern (Miglio et al. 2008), which typically manifest themselves as periodic dips in the spacings. The periodicity of these non-uniform variations is strongly linked to the location of the chemical gradient inside the star, while their amplitude is related to the steepness of the gradient.

Stellar rotation leads to shifts in the pulsation frequencies, so that spacing patterns of retrograde modes $(m<0)$ in a 
rotating star have an overall upward slope, while patterns of zonal and prograde modes $(m>0)$ have a downward slope (Bouabid et al. 2013). In addition, rotational mixing, like other extra mixing processes, affects the chemical gradients in the stellar interior, typically reducing the steepness of the gradients, which leads to smaller non-uniform variations in the observed patterns (Bouabid et al. 2013). Furthermore, stellar rotation may also affect the propagation cavity of the gravito-inertial modes. While pure $g$-modes cannot propagate into a convective region, gravito-inertial modes in a rotating star can. This leads to an additional shift of the observed pulsation frequencies (Prat et al. 2017).

Thanks to the high-precision photometric observations of space missions such as Kepler (Koch et al. 2010), such period spacing patterns were recently found in some $\gamma$ Doradus stars (e.g. Chapellier et al. 2012; Kurtz et al. 2014; Bedding et al. 2015; Van Reeth et al. 2015b; Keen et al. 2015; Saio et al. 2015). They have since been used to probe the rotation rates (e.g. Murphy et al. 2016; Van Reeth et al. 2016; Ouazzani et al. 2017; Sowicka et al. 2017; Guo et al. 2017) and the nature of the mixing processes (e.g. Schmid \& Aerts 2016) in the deep interior of these stars. This provides us with an opportunity to independently evaluate the rotational mixing and angular momentum transport mechanisms inside intermediate-mass stars. Previous studies of the internal rotation profiles using space-based photometric observations have revealed shortcomings in the existing theory for these mechanisms. The observed surface-to-core rotation rates in red giants are orders of magnitude higher than predicted by theory (e.g. Marques et al. 2013; Cantiello et al. 2014), and Triana et al. (2015) have even found a counterrotation in the B-type star KIC 10526294. Internal gravity waves have been proposed as a mechanism to explain these observations (Rogers et al. 2013; Rogers 2015).

The star 43 Cygni (HD 195068/9, V 2121 Cygni, HR 7828) was originally identified as a possible RR Lyrae star from HIPPARCOS photometry (Perryman et al. 1997). Handler (1999) indicated the possible $\gamma$ Doradus nature of $43 \mathrm{Cyg}$. From observed asymmetries in the line profiles, Fekel et al. (2003) concluded that 43 Cygni is either a binary or a pulsator. Gerbaldi et al. (2007) verified that 43 Cyg is a single star from TD1 UV observations. Henry et al. (2005) identified the star as a (single) $\gamma$ Doradus pulsator and detected three main frequencies at $f_{1}=1.25054 \mathrm{~d}^{-1}, f_{2}=1.29843 \mathrm{~d}^{-1}$, and $f_{3}=0.96553 \mathrm{~d}^{-1}$, using photometric time series in the Johnson $B$ and $V$ filters. Using 230 spectra obtained over two years, Jankov et al. (2006) studied the line profile variations, detected an additional frequency, and attempted a first spectroscopic mode identification. The authors reported a frequency at $1.61 \mathrm{~d}^{-1}$ to be an $\ell=5( \pm 1)$ and $m=4( \pm 1)$ and the frequency at $1.25 \mathrm{~d}^{-1}$, which is $f_{1}$ of Henry et al. (2005), to be an $\ell=4( \pm 1)$ and $m=3( \pm 1)$ mode. Cuypers et al. (2009) confirmed the presence of the previously identified pulsation frequencies using photometric time series in the Geneva system. In the literature, 43 Cygni is reported to have an effective temperature, $T_{\text {eff }}$, of $7300 \pm 250 \mathrm{~K}$ and a $\log g$ of $4.35 \pm 0.14 \mathrm{cms}^{-2}$ (David \& Hillenbrand 2015); its projected rotational velocity, $v \sin i$, was determined to be $44 \mathrm{~km} \mathrm{~s}^{-1}$ (e.g. Fekel et al. 2003).

As 43 Cygni has a $V$ magnitude of 5.74, it is at the fainter end of the magnitude range observable with BRITE-Constellation (Weiss et al. 2014). In the present study we describe the BRITEConstellation observations of 43 Cygni and conduct a detailed asteroseismic investigation of the pulsation periods and their spacings with the aim to constrain the star's near-core rotation. We also use new high-resolution spectroscopy with a high signal-to-noise ratio to redetermine the fundamental parameters and for a first investigation of the chemical abundances of 43 Cygni.

\section{Observations and data reduction}

\subsection{BRITE-Constellation}

BRITE-Constellation $^{1}$ is a fleet of five nano-satellites measuring light variations in stars typically brighter than $V=5 \mathrm{mag}$. Each $20 \mathrm{~cm}$ cube satellite carries a telescope with an aperture of $3 \mathrm{~cm}$ that feeds an uncooled CCD (Weiss et al. 2014). Three of the BRITE satellites, that is, BRITE-Toronto (BTr), Uni-BRITE (UBr), and BRITE-Heweliusz (BHr), carry a custom-defined red filter $(550-700 \mathrm{~nm})$, and two satellites, that is, BRITEAustria (BAb) and BRITE-Lem (BLb), carry a custom-defined blue filter (390-460 nm). More details on the detectors and prelaunch and in-orbit tests are described by Pablo et al. (2016). Popowicz et al. (2017) described the pipeline that processes the observed images, yielding the instrumental magnitudes that are delivered to the users.

BRITE-Constellation observes large fields with typically 15 to 20 stars brighter than $V=6$ mag including at least three targets brighter than $V=3$ mag. Each field is observed at least 15 min for each $\sim 100$-min orbit for up to half a year (Weiss et al. 2014).

BRITE-Constellation obtained observations of the Cygnus-II field from June 1 to November 25, 2015. The star 43 Cygni was observed for only 13 days with BRITE-Lem (BLb) in the blue filter and for 156 days with BRITE-Toronto (BTr) in the red filter, both in chopping mode, where the position of the target star within the CCD plane is constantly alternated between two positions about 20 pixels apart on the CCD. This procedure was adopted to mitigate the impact of high dark current in CCDs. A detailed description of this technique is given in Popowicz et al. (2017). As the blue data set from BLb did not have sufficient quality for an asteroseismic analysis, we omitted it from our further investigation.

The BRITE-Constellation data of 43 Cyg were corrected for instrumental effects according to the iterative procedure described by Pigulski et al. (2016). This procedure was, however, modified to include two-dimensional decorrelations. Since 43 Cyg shows variability with large amplitude, decorrelations were made using residuals from a fit consisting of a sum of sinusoidal terms. For $43 \mathrm{Cyg}$, the model consisted of five terms: four modes with frequencies $1.250 \mathrm{~d}^{-1}, 1.288 \mathrm{~d}^{-1}, 0.963 \mathrm{~d}^{-1}$, and $1.516 \mathrm{~d}^{-1}$, plus a combination frequency $\left(1.250 \mathrm{~d}^{-1}-0.963 \mathrm{~d}^{-1}=\right.$ $0.287 \mathrm{~d}^{-1}$ ). This model was used for all BTr setups. The fit was recalculated at each step of the decorrelation. The whole procedure included outlier rejection, 1D and 2D decorrelations, and removal of corrupted orbits (e.g. affected by poor stability of the satellite). Decorrelations, both 1D and 2D, were made using parameters provided in Data Release 3 (see Popowicz et al. 2017, or the BRITE Public Data Archive ${ }^{2}$ for the explanation of parameters) and a given satellite orbital phase as an additional parameter. The decorrelation was made sequentially, allowing for multiple decorrelations with the same parameter (or a pair of parameters). At each step, the parameter showing the strongest correlation was chosen for correction. The strength of a correlation was defined as the degree of reduction in variance due to decorrelation with a given parameter (for 1D) or a pair of parameters (for 2D). The iterations were stopped when all correlations

\footnotetext{
http: //www . brite-constellation . at

https://brite.camk.edu.pl/pub/index.html
} 

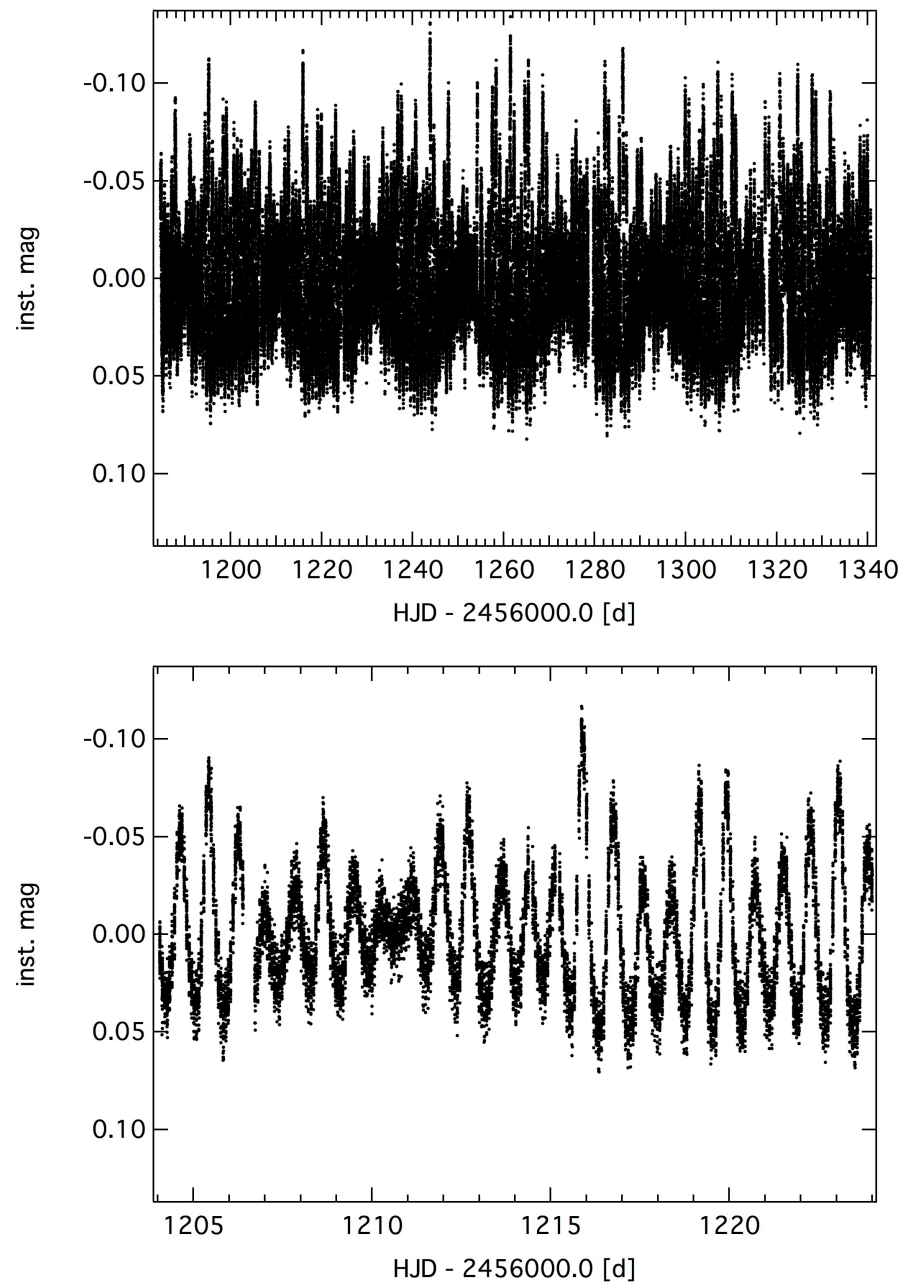

Fig. 1. Complete BRITE-Toronto light curve of 43 Cygni (top) and zoom into a 20-day subset (bottom).

(both 1D and 2D) resulted in a variance reduction smaller than 0.05 per cent. The whole procedure was run independently for each setup. Outliers and the worst orbits were rejected at least twice during the whole procedure. After decorrelating the data, the blue and red data were separately combined, taking the mean magnitude offsets between the setups into account.

The complete BTr light curve of 43 Cygni consists of 98481 data points and is shown in the top panel of Fig. 1, while the bottom panel shows a zoom into a 20 -day subset.

\subsection{HERMES spectra}

We obtained ten high-resolution spectra with high signal-tonoise ratio $(\mathrm{S} / \mathrm{N})$ for 43 Cygni using the HERMES spectrograph $(R=85,000, \lambda=377-900 \mathrm{~nm}$, Raskin et al. 2011) at the $1.2 \mathrm{~m}$ Mercator telescope (Observatorio del Roque de los Muchachos, La Palma, Canary Islands, Spain). The observations were taken during seven individual nights between August 4 and 15, 2016, with a typical exposure time of $600 \mathrm{~s}$ and an $\mathrm{S} / \mathrm{N}$ higher than 150 per pixel. The individual spectra were reduced with the most recent version of the HERMES pipeline (release 6) and normalized by fitting a low-order polynomial to carefully selected continuum points. The resulting combined normalized spectrum has an $\mathrm{S} / \mathrm{N}$ of 590 calculated from 5820 to $5821 \AA$ and was used to determine the fundamental parameters and abundances for

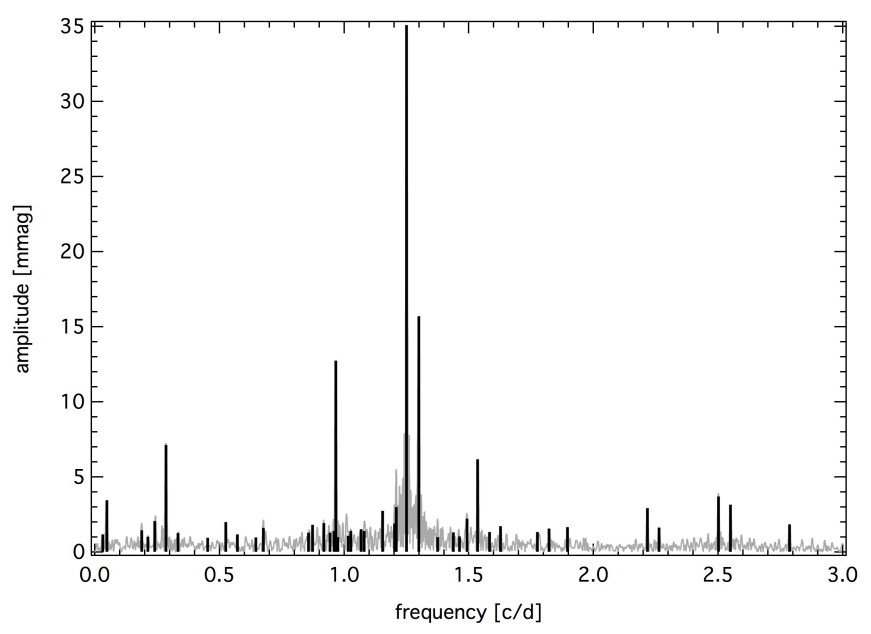

Fig. 2. Original amplitude spectrum of 43 Cygni (gray) where the identified pulsation frequencies are marked in black.

43 Cygni. With our spectroscopic data, we can also verify previous reports (e.g. Henry et al. 2005; Gerbaldi et al. 2007) that 43 Cyg is a single star.

\section{Frequency analysis}

The frequency analysis of the BRITE photometric time series was performed using an iterative pre-whitening method based on the Lomb-Scargle periodogram, which is described by Van Reeth et al. (2015a). Frequencies are identified to be significant if they exceed 3.9 times the local noise level in the Fourier domain. Frequency errors are calculated using the method by Schwarzenberg-Czerny (2003), which is based on the statistical errors resulting from a non-linear least-squares fit corrected for the correlated nature of the data. Both methods have previously been successfully applied to $\gamma$ Doradus pulsation (e.g. Van Reeth et al. 2015b).

In total, we detected 43 intrinsic frequencies with an $\mathrm{S} / \mathrm{N}$ higher than 3.9 in a range from 0 to $2.78 \mathrm{~d}^{-1}$ that are caused by $\gamma$ Doradus-type pulsations (see Fig. 2 and Table 1). We can also confirm the previously identified frequencies at $1.25054 \mathrm{~d}^{-1}$, $1.29843 \mathrm{~d}^{-1}, 0.96553 \mathrm{~d}^{-1}$, and $1.61 \mathrm{~d}^{-1}$ reported by Henry et al. (2005) and Jankov et al. (2006).

\section{Spectroscopic analysis}

Spectroscopic analysis of $43 \mathrm{Cyg}$ was performed using the LLMODELS model atmosphere code (Shulyak et al. 2004), the VALD database for atomic line parameters (Kupka et al. 1999), SYNTH3 (Kochukhov 2007) for the computation of synthetic spectra, and an updated version of the SME (Spectroscopy Made Easy, version 474) software package (Valenti \& Piskunov 1996; Piskunov \& Valenti 2017).

SME allows us to derive effective temperature, surface gravity, overall metallicity, individual element abundances, and the microturbulent, macroturbulent, rotational, and radial velocities of a star by fitting synthetic spectra to those observed. Spectral synthesis calculations may be performed for different grids of model atmospheres and are interpolated between the grid nodes. We used a model grid calculated with the LLMODELS stellar model atmosphere code for a microturbulent velocity 
Table 1. Pulsation frequencies, periods, amplitudes, and $\mathrm{S} / \mathrm{N}$ values for 43 Cyg obtained from the BRITE data using the iterative pre-whitening procedure and sorted by increasing frequency.

\begin{tabular}{|c|c|c|c|c|c|}
\hline \# & $\begin{array}{c}\text { Frequency } \\
\qquad\left[\mathrm{d}^{-1}\right]\end{array}$ & $\begin{array}{l}\text { Period } \\
{[\mathrm{d}]}\end{array}$ & $\begin{array}{c}\text { Amp } \\
{[\mathrm{mmag}]}\end{array}$ & $S / N$ & Comment \\
\hline 1 & $0.03163(16)$ & $31.61626(16345)$ & 1.192 & 4.69 & $f_{32}-f_{31}$ \\
\hline 2 & $0.04731(8)$ & $21.13692(3565)$ & 3.449 & 9.07 & $f_{28}-f_{27}$ \\
\hline 3 & $0.18754(14)$ & $5.33210(403)$ & 1.466 & 4.21 & $f_{30}-f_{27}$ \\
\hline 4 & $0.21185(19)$ & $4.72034(423)$ & 1.013 & 4.04 & $f_{31}-f_{27}$ \\
\hline 5 & $0.24033(11)$ & $4.16099(198)$ & 2.073 & 6.31 & $f_{32}-f_{27}$ \\
\hline 6 & $0.28474(4)$ & $3.51203(5)$ & 7.146 & 16.56 & $f_{33}-f_{27}=f_{27}-f_{18}$ \\
\hline 7 & $0.33332(16)$ & $3.00013(142)$ & 1.281 & 4.90 & $f_{34}-f_{27}$ \\
\hline 8 & $0.45208(20)$ & 2.21202(98) & 0.947 & 3.94 & $f_{33}-f_{23}$ \\
\hline 9 & $0.52487(12)$ & $1.90523(43)$ & 1.997 & 6.17 & $f_{36}-f_{27}$ \\
\hline 10 & $0.57092(17)$ & $1.75155(51)$ & 1.185 & 4.90 & $f_{37}-f_{27}$ \\
\hline 11 & $0.64546(20)$ & $1.54928(47)$ & 0.974 & 3.90 & $f_{38}-f_{27}$ \\
\hline 12 & $0.67609(14)$ & $1.47908(30)$ & 1.609 & 5.56 & \\
\hline 13 & $0.85685(15)$ & $1.16707(21)$ & 1.285 & 4.84 & $f_{36}-f_{15}=f_{37}-f_{18}$ \\
\hline 14 & $0.87263(12)$ & $1.14596(16)$ & 1.810 & 5.90 & $f_{38}-f_{21}$ \\
\hline 15 & $0.91798(12)$ & $1.08935(14)$ & 1.933 & 6.02 & \\
\hline 16 & $0.94311(15)$ & $1.06032(17)$ & 1.289 & 4.70 & \\
\hline 17 & $0.95917(15)$ & $1.04257(16)$ & 1.418 & 5.56 & \\
\hline 18 & $0.96593(3)$ & $1.03527(3)$ & 12.758 & 26.60 & previously identified $[1,2]$ \\
\hline 19 & $0.97504(19)$ & $1.02559(20)$ & 0.994 & 3.91 & \\
\hline 20 & $1.01565(18)$ & $0.98459(17)$ & 1.086 & 4.20 & pattern \\
\hline 21 & $1.02591(14)$ & $0.97475(14)$ & 1.404 & 4.91 & pattern \\
\hline 22 & $1.06732(14)$ & $0.93693(12)$ & 1.521 & 5.41 & pattern \\
\hline 23 & $1.08058(15)$ & $0.92543(13)$ & 1.407 & 4.93 & pattern \\
\hline 24 & $1.15385(9)$ & $0.86667(7)$ & 2.750 & 7.47 & pattern \\
\hline 25 & $1.20222(12)$ & $0.83179(8)$ & 1.915 & 5.93 & $f_{38}-f_{15}$ \\
\hline 26 & $1.20847(9)$ & $0.82749(6)$ & 2.998 & 8.11 & pattern \\
\hline 27 & $1.25043(2)$ & $0.79973(1)$ & 35.094 & 48.07 & pattern; previously identified $[1,2]$ \\
\hline 28 & $1.29856(3)$ & $0.77008(2)$ & 15.699 & 29.54 & pattern; previously identified $[1,2]$ \\
\hline 29 & $1.37508(19)$ & $0.72723(10)$ & 1.001 & 4.18 & pattern \\
\hline 30 & $1.43765(15)$ & $0.69558(7)$ & 1.321 & 4.94 & pattern \\
\hline 31 & $1.46167(19)$ & $0.68415(9)$ & 1.036 & 4.18 & pattern \\
\hline 32 & $1.49255(11)$ & $0.66999(5)$ & 2.219 & 6.53 & pattern \\
\hline 33 & $1.53544(5)$ & $0.65128(2)$ & 6.178 & 15.62 & pattern \\
\hline 34 & $1.58267(15)$ & $0.63184(6)$ & 1.340 & 4.87 & pattern \\
\hline 35 & $1.62701(13)$ & $0.61463(5)$ & 1.727 & 5.49 & pattern; previously identified $[1,2]$ \\
\hline 36 & $1.77541(15)$ & $0.56325(5)$ & 1.344 & 5.07 & pattern \\
\hline 37 & $1.82161(13)$ & $0.54896(4)$ & 1.570 & 5.38 & pattern \\
\hline 38 & $1.89567(13)$ & $0.52752(4)$ & 1.662 & 5.58 & pattern \\
\hline 39 & $2.21623(9)$ & $0.45122(2)$ & 2.930 & 8.40 & $f_{18}+f_{27}$ \\
\hline 40 & $2.26298(13)$ & $0.44189(3)$ & 1.626 & 5.88 & $f_{18}+f_{28}$ \\
\hline 41 & $2.50095(8)$ & $0.39985(1)$ & 3.717 & 10.24 & $2 f_{27}$ \\
\hline 42 & $2.54918(8)$ & $0.39228(1)$ & 3.157 & 10.06 & $f_{27}+f_{28}$ \\
\hline 43 & $2.78583(12)$ & $0.35896(2)$ & 1.851 & 7.10 & $f_{27}+f_{29}$ \\
\hline
\end{tabular}

Notes. References are 1: Henry et al. (2005) and 2: Jankov et al. (2006). Pulsations indicated with the comment "pattern" are part of the periodspacing pattern discussed in Sect. 5.

$v_{\text {mic }}=2.0 \mathrm{~km} \mathrm{~s}^{-1}$, which ranges from 4500 to $22000 \mathrm{~K}$ in effective temperature, from 2.5 to 5.0 dex in surface gravity, and from -0.8 to 0.8 dex in metallicity (see Table 5 of Tkachenko et al. 2012). The corresponding steps are $0.1 \mathrm{dex}$ in $\log g, 0.1 \mathrm{dex}$ in metallicity, $100 \mathrm{~K}$ in the effective temperature region from 4500 to $10000 \mathrm{~K}$ and $250 \mathrm{~K}$ for higher $T_{\text {eff }}$ values.

To fit the synthetic spectra, we followed an approach described for example by Ryabchikova et al. (2016) and used the following spectral regions: 4167-4511 $\mathrm{A}, 4485-4590 \AA$,

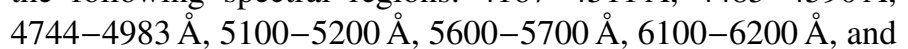
6335-6765 $\AA$. These ranges include the three Balmer lines $\mathrm{H} \alpha$,
$\mathrm{H} \beta$, and $\mathrm{H} \gamma$. For a comparison and consistency check, we also used the complete spectral range from 4150-6800 $\mathrm{A}$.

As starting values for our computations, we used the values of $T_{\text {eff }}=7300 \mathrm{~K}$ and $\log g=4.35$ provided by David \& Hillenbrand (2015) and $v \sin i=44 \mathrm{~km} \mathrm{~s}^{-1}$ from Fekel et al. (2003). We used four different approaches to investigate the fundamental parameters for $43 \mathrm{Cyg}$ : (i) we used only the three Balmer lines as input for SME, (ii) we used the four spectral regions without the Balmer lines, (iii) we used all above mentioned regions at the same time, and (iv) the complete spectral range from $4150-6800 \AA$. The corresponding solutions 
Table 2. Stellar parameters obtained with SME.

\begin{tabular}{ll}
\hline \hline$T_{\mathrm{eff}}$ & $7150 \pm 150 \mathrm{~K}$ \\
$\log g$ & $4.2 \pm 0.6 \mathrm{dex}$ \\
$v \sin i$ & $44 \pm 4 \mathrm{~km} \mathrm{~s}^{-1}$ \\
{$[\mathrm{Fe} / \mathrm{H}]$} & $-0.05 \pm 0.08 \mathrm{dex}$ \\
$v_{\text {micro }}$ & $2.99 \pm 0.37 \mathrm{~km} \mathrm{~s}^{-1}$ \\
\hline
\end{tabular}

are very similar to each other: for $T_{\text {eff }}, \log g$, and metallicity $[\mathrm{Fe} / \mathrm{H}]$, we find (i) $7141 \mathrm{~K} / 4.11 /-0.21$, (ii) $7308 \mathrm{~K} / 4.29 /-0.05$, (iii) $7144 / 4.15 /-0.18$, and (iv) $7154 \mathrm{~K} / 4.24 /-0.24$. All four approaches find $v \sin i$ values of $44 \pm 4 \mathrm{~km} \mathrm{~s}^{-1}$ and that the microturbulent velocity, $v_{\text {mic }}$, varies around a value of $2.9 \mathrm{~km} \mathrm{~s}^{-1}$.

In the present temperature range, the hydrogen lines are sensitive to changes in both $T_{\text {eff }}$ and $\log g$. We therefore used the average of the results from approaches (i), (iii), and (iv), which include $\mathrm{H} \alpha, \mathrm{H} \beta$, and $\mathrm{H} \gamma$ as our final values of $T_{\text {eff }}$ and $\log g$. Hence, we adopted the final parameters as $T_{\mathrm{eff}}=7150 \pm 150 \mathrm{~K}$, $\log g=4.2 \pm 0.6, v \sin i=44 \pm 4 \mathrm{~km} \mathrm{~s}^{-1}$, and $v_{\text {mic }}=2.99 \pm$ $0.37 \mathrm{~km} \mathrm{~s}^{-1}$ (Table 2). Figure 3 shows a comparison between the observed and calculated line profiles of $\mathrm{H} \alpha, \mathrm{H} \beta$, and $\mathrm{H} \gamma$.

We also conducted a detailed study of the star's individual chemical abundances using the SME software. We treated each element individually and calculated the atmospheric abundances in local thermal equilibrium (LTE), except for $\mathrm{O}, \mathrm{Na}, \mathrm{Ca}$, and $\mathrm{Ba}$, for which we used a non-LTE analysis based on the procedure of Piskunov et al. (2017). Just as we have found for the global metallicity of the star, the individual abundances of all analysed elements agree with the solar values within the quoted error bars (see Table 3 and Fig. 4). From this analysis, we adopt a final value of $[\mathrm{Fe} / \mathrm{H}]$ of $-0.05 \pm 0.08$ for $43 \mathrm{Cyg}$.

\section{Asteroseismic inference}

\subsection{Identification of a period-spacing pattern}

The extracted pulsation frequencies were subsequently converted into periods and analysed to search for period spacing patterns. It is well known that because of the typical low noise level, the classical $\mathrm{S} / \mathrm{N}$ criterion $S / N \geq 4$ is unsuitable for the application of iterative pre-whitening on space-based data. As for example discussed by Balona (2014) and Baran et al. (2015), the probability of including spurious extracted frequencies when studying space-based data is higher than for ground-based data with a lower $\mathrm{S} / \mathrm{N}$. In the case of the latter, the classical stop criterion for iterative pre-whitening is typically fulfilled earlier during the data analysis, so that fewer frequencies are extracted. Spurious extracted frequencies may be caused by the attempted pre-whitening of unresolved pulsation frequencies, for instance, and/or the residuals of imperfect preceding prewhitening. Hence, to facilitate the search for period-spacing patterns in the data, we used the evaluation criterion defined by Van Reeth et al. (2015a) to help assess the reliability of the extracted signal, and classified the pulsation periods into nine different groups accordingly. In this evaluation, we compared the amplitudes of the extracted pulsations with the local value of the Lomb-Scargle periodogram at that pulsation period. The relative difference between these two values was used as a measure for the effect of the preceding iterative pre-whitening.

This resulted in the detection of one period-spacing pattern, which is shown in Fig. 5 and listed in Table 1. The majority of the extracted pulsation frequencies, which are not part of this pattern, were found to be combination frequencies. To identify
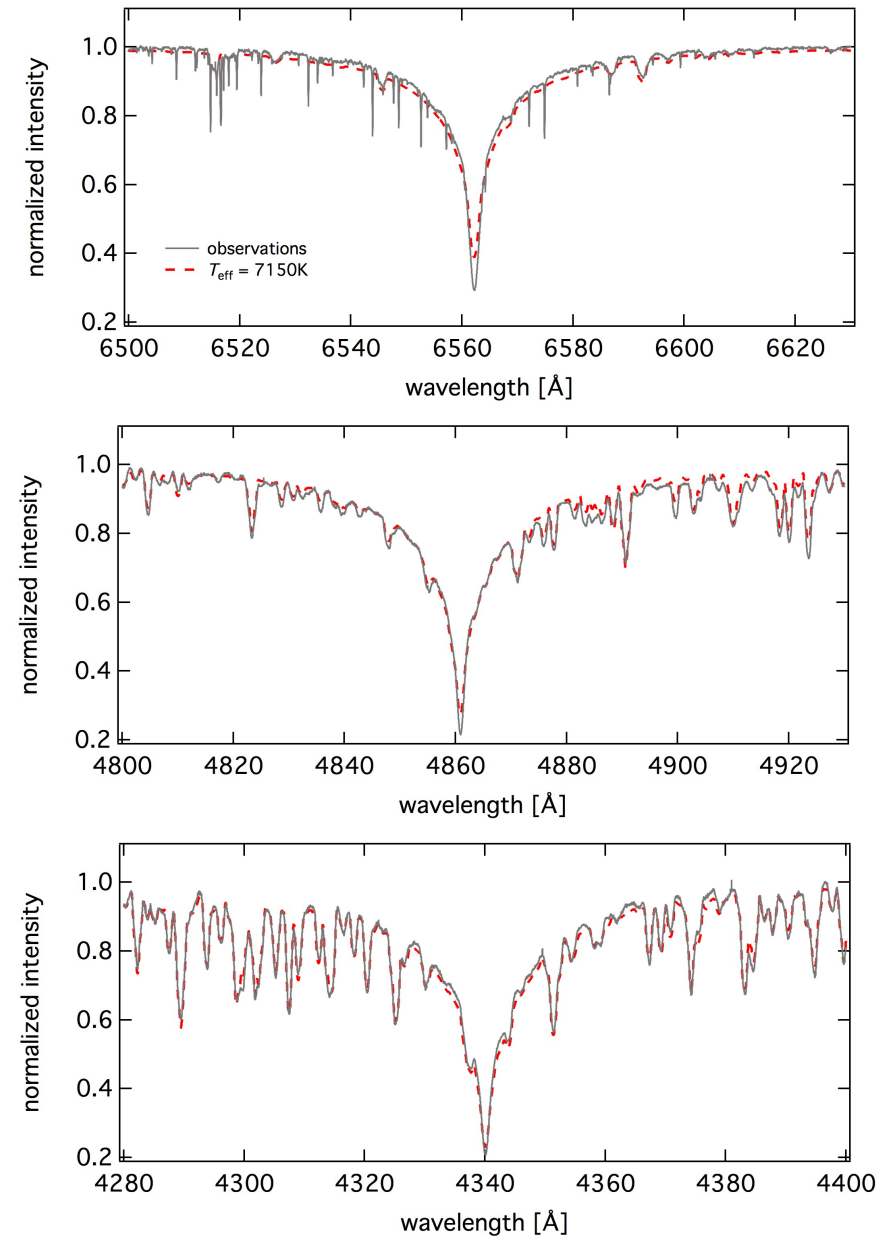

Fig. 3. Region of the $\mathrm{H} \alpha$ (top), $\mathrm{H} \beta$ (middle) and $\mathrm{H} \gamma$ (bottom) lines: the observed spectrum is shown in black and the calculated synthetic spectrum with the final adopted parameters of $T_{\text {eff }}=7150 \mathrm{~K}$ and $\log g=$ 4.2 in red.

possible linear combinations, we used the frequency resolution of the data set, that is, $1 / T=0.006 \mathrm{~d}^{-1}$, as a tolerance margin. This is also listed in Table 1. In addition, the other detected pulsation frequencies could also be isolated pulsations, possibly with mode identifications $(l, m)$ different from that of the detected pattern, or in the case of some of the lower-amplitude signal, numerical artefacts originating from the iterative prewhitening routine.

Although the detected pattern is relatively short and has gaps, there is a clear downward slope, caused by the stellar rotation. The pattern also contains strong non-uniform variations, indicative of a chemical gradient in the deep stellar interior. This is especially obvious when the pattern is compared to previously detected patterns, as shown in Fig. 6. While a detailed asteroseismic modelling would require a more extensive period-spacing pattern, and thus, a significantly longer time base of observations, the current data allow for a basic asteroseismic evaluation. We can (i) determine the mode identification $(l, m)$ of the pulsation modes in the period-spacing pattern and compute the asymptotic period spacing $\Delta \Pi_{l=1}$ and near-core rotation rate $f_{\text {rot }}$ from the observed period-spacing pattern (see Sect. 5.2); and (ii) evaluate the consistency between the obtained spectroscopic properties of the star and the derived asymptotic period-spacing and near-core rotation rate using a suite of MESA/GYRE models (see Sect. 5.3). 
Table 3. Atmospheric abundances for 43 Cygni with the error estimates based on the internal scattering from the number of the analysed lines compared to solar values.

\begin{tabular}{lcc}
\hline \hline Element & $\begin{array}{c}43 \mathrm{Cyg} \\
\log \left(N_{\mathrm{el}} / N_{\text {tot }}\right)\end{array}$ & $\begin{array}{c}\text { Sun } \\
\log \left(N_{\mathrm{el}} / N_{\text {tot }}\right)\end{array}$ \\
\hline $\mathrm{C}$ & $-3.59 \pm 0.36$ & -3.52 \\
$\mathrm{O}$ & $-3.23 \pm 0.07$ & -3.21 \\
$\mathrm{Na}$ & $-5.48 \pm 0.04$ & -5.71 \\
$\mathrm{Mg}$ & $-4.39 \pm 0.14$ & -4.46 \\
$\mathrm{Si}$ & $-4.38 \pm 0.25$ & -4.49 \\
$\mathrm{Ca}$ & $-5.63 \pm 0.08$ & -5.68 \\
$\mathrm{Sc}$ & $-8.73 \pm 0.09$ & -8.87 \\
$\mathrm{Ti}$ & $-7.02 \pm 0.10$ & -7.02 \\
$\mathrm{Cr}$ & $-6.40 \pm 0.29$ & -6.37 \\
$\mathrm{Mn}$ & $-6.87 \pm 0.23$ & -6.65 \\
$\mathrm{Fe}$ & $-4.56 \pm 0.08$ & -4.54 \\
$\mathrm{Ni}$ & $-5.78 \pm 0.28$ & -5.79 \\
$\mathrm{Cu}$ & $-7.84 \pm 0.22$ & -7.83 \\
$\mathrm{Y}$ & $-9.81 \pm 0.14$ & -9.80 \\
$\mathrm{Ba}$ & $-9.73 \pm 0.08$ & -9.91 \\
\hline
\end{tabular}

Notes. Abundances of $\mathrm{O}, \mathrm{Na}, \mathrm{Ca}$, and $\mathrm{Ba}$ were calculated in non-LTE based on Piskunov et al. (2017), all other elements in LTE. For comparison, the last column gives the abundances of the solar atmosphere calculated by Asplund et al. (2009).

\subsection{Mode-identification and near-core rotation rate}

In the first part of our analysis, we subsequently fitted a modelspacing pattern to these observations to yield a mode identification for these pulsations and derive the near-core rotation rate. In this model, we ignored the non-uniform variations in period-spacing patterns caused by chemical gradients, and assumed a rigidly rotating chemically homogeneous star. We used the asymptotic equidistant period-spacing pattern derived by Tassoul (1980) and included the effects of rotation using the traditional approximation (e.g. Eckart 1960; Lee \& Saio 1987 1997). The fit of the model pattern to the observations was evaluated using a $\chi^{2}$-statistic. We refer to Van Reeth et al. (2016) for a detailed description and validation of this method.

For the evaluation of this basic model pattern, we excluded the pulsation periods that were strongly affected by the chemical gradients in the stellar interior, at $0.55 \mathrm{~d}$ and $0.68 \mathrm{~d}$. A chemical gradient inside the star causes trapping of pulsation modes (Miglio et al. 2008), and as we can clearly see in Fig. 5, these two pulsation periods are responsible for the dips in the detected period-spacing pattern. The traditional approximation was implemented using the TAR module of the 1D stellar pulsation code GYRE v5.0 (Townsend \& Teitler 2013). We identified the pulsations as prograde dipole modes and found a near-core rotation frequency $f_{\text {rot }}=0.56_{-0.14}^{+0.12} \mathrm{~d}^{-1}$ and an asymptotic spacing $\Delta \Pi_{l=1}=2970_{-570}^{+700} \mathrm{~s}$. This is in full agreement with the results of previous studies of $\gamma$ Dor stars in the literature (e.g. Van Reeth et al. 2016; Ouazzani et al. 2017). This result is also shown in Fig. 7.

\subsection{Comparison between spectroscopic and asteroseismic properties}

In the second part of this analysis, we made a consistency check between the spectroscopic and photometric parameters, that is, $T_{\text {eff }}, \log g,[M / H], \Delta \Pi_{l=1}$, and $f_{\text {rot }}$, using stellar

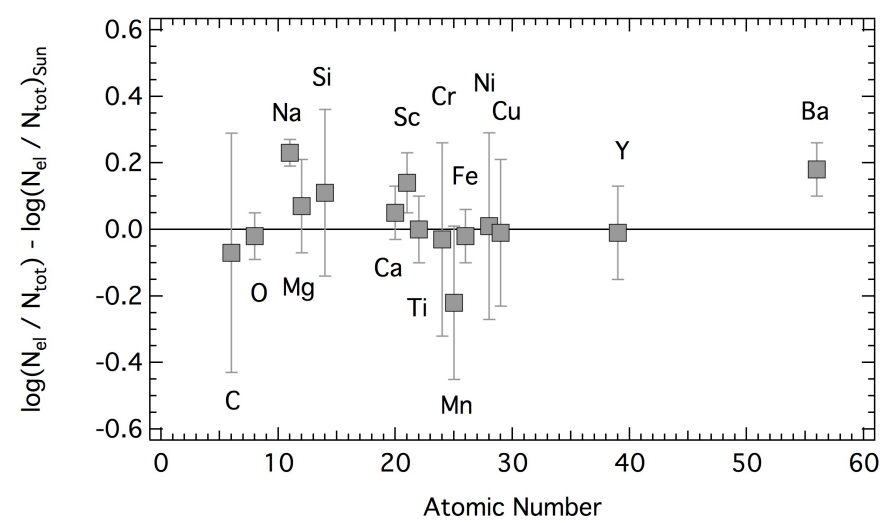

Fig. 4. Atmospheric abundances of 43 Cyg relative to the Sun.

models computed with the stellar evolution code MESA v7385 (Paxton et al. 2011, 2013, 2015). We found that, in part because of the large error margins on the different parameters, the derived parameter values are entirely consistent. This is illustrated in the Kiel diagram in Fig. 8. The stellar evolution tracks in this figure were computed assuming solar metallicity, using the solar abundances derived by Asplund et al. (2009). The convection was treated using the mixing-length theory, with $\alpha_{\text {MLT }}$ equal to 1.8 , and the models include exponential core overshooting, assuming $f_{\text {ov }}$ equal to 0.015 . These parameter values were chosen to be close to typical values found in the literature for mainsequence $g$-mode pulsators (e.g. Moravveji et al. 2015, 2016; Schmid \& Aerts 2016).

\subsection{Morphology of the period-spacing pattern and diffusive mixing}

In addition, the morphology of the observed pattern also has some interesting characteristics. The large dips in the observed period-spacing pattern point to the presence of a significant chemical gradient in the stellar interior. The relative amplitude of the dips in this pattern is comparable to those of the largest dips previously observed in period-spacing patterns of $\gamma$ Dor stars (e.g. Bedding et al. 2015; Van Reeth et al. 2015b). This is a possible indication that the amount of chemical mixing in this star is on the lower end of what can be expected for a typical $\gamma$ Dor star.

Hence, we have made an additional consistency check, whereby we qualitatively compared the observed data with two theoretical patterns that were calculated with the stellar pulsation code GYRE, using stellar models computed with the stellar evolution code MESA as input. This is illustrated in the bottom panel of Fig. 5. These two models were selected in agreement with the values of $T_{\text {eff }}, \log g$, and $\Delta \Pi_{l=1}$ within the $1 \sigma$ confidence intervals. They have a stellar mass of $1.6 M_{\odot}$, solar metallicity, a convective core exponential overshoot of 0.015 , and a central hydrogen fraction $X_{\mathrm{c}}$ of 0.36 . The two models have a constant extra diffusive mixing $D_{\text {mix }}$ in the radiative region of $0 \mathrm{~cm}^{2} \mathrm{~s}^{-1}$ and $1 \mathrm{~cm}^{2} \mathrm{~s}^{-1}$, respectively. In these models, the extra diffusive mixing reduces the steepness of the chemical gradients in the stellar interior. It seems that for these models, an extra mixing $D_{\text {mix }}$ of $1 \mathrm{~cm}^{2} \mathrm{~s}^{-1}$ is an upper limit for $43 \mathrm{Cyg}$. Thus, we speculate that one or more steep chemical gradients are present in the interior of $43 \mathrm{Cyg}$. Detailed asteroseismic modelling is required to accurately determine the amount of extra mixing inside the star and evaluate the validity of these models. However, this is beyond the scope of this paper. 
K. Zwintz et al.: Constraining the near-core rotation of 43 Cygni

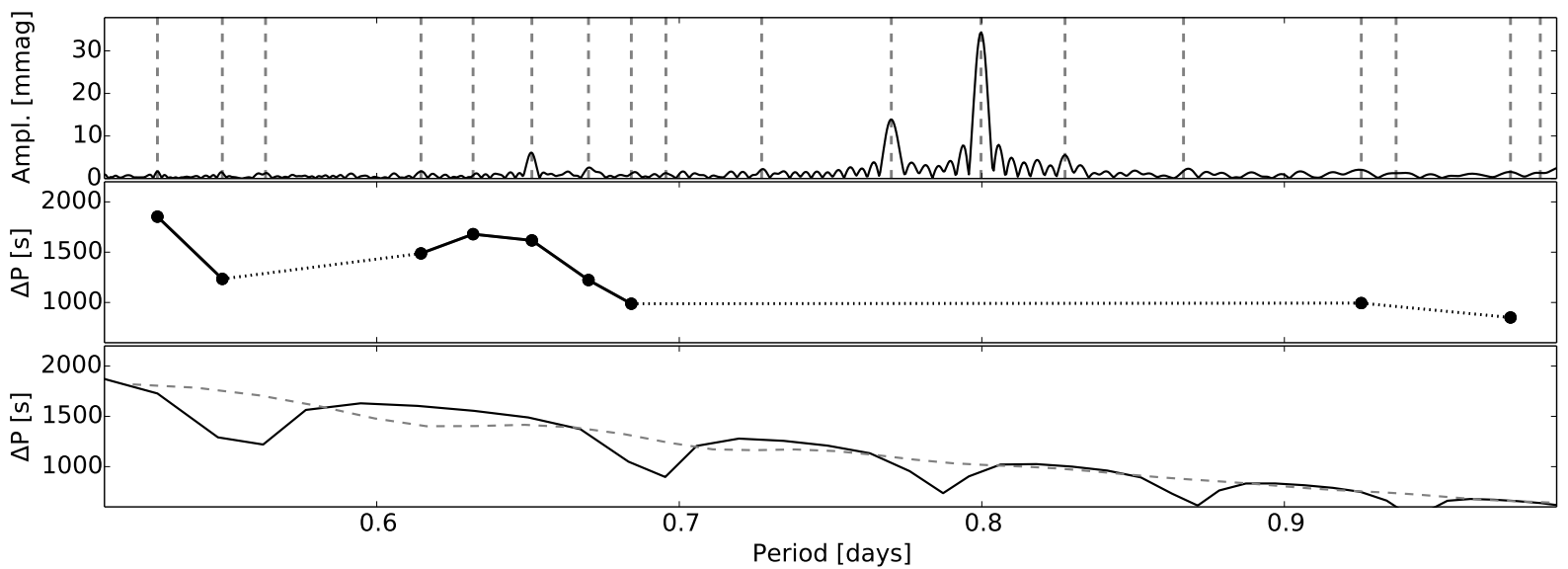

Fig. 5. Top: part of the Fourier spectrum where the period-spacing pattern was found, with the pulsation periods of the pattern marked by dashed lines. Middle: detected period-spacing pattern. Bottom: two model period-spacing patterns, for $M=1.6 M_{\odot}$, solar metallicity, $\alpha_{\mathrm{MLT}}=1.8$, $f_{\text {ov }}=0.015 H_{\mathrm{p}}$, and $X_{\mathrm{c}}=0.36$. The models have a constant extra diffusive mixing $D_{\text {mix }}$ in the radiative region of $0 \mathrm{~cm}^{2} \mathrm{~s}^{-1}$ (full black) and $1 \mathrm{~cm}^{2} \mathrm{~s}^{-1}$ (dashed grey), respectively.

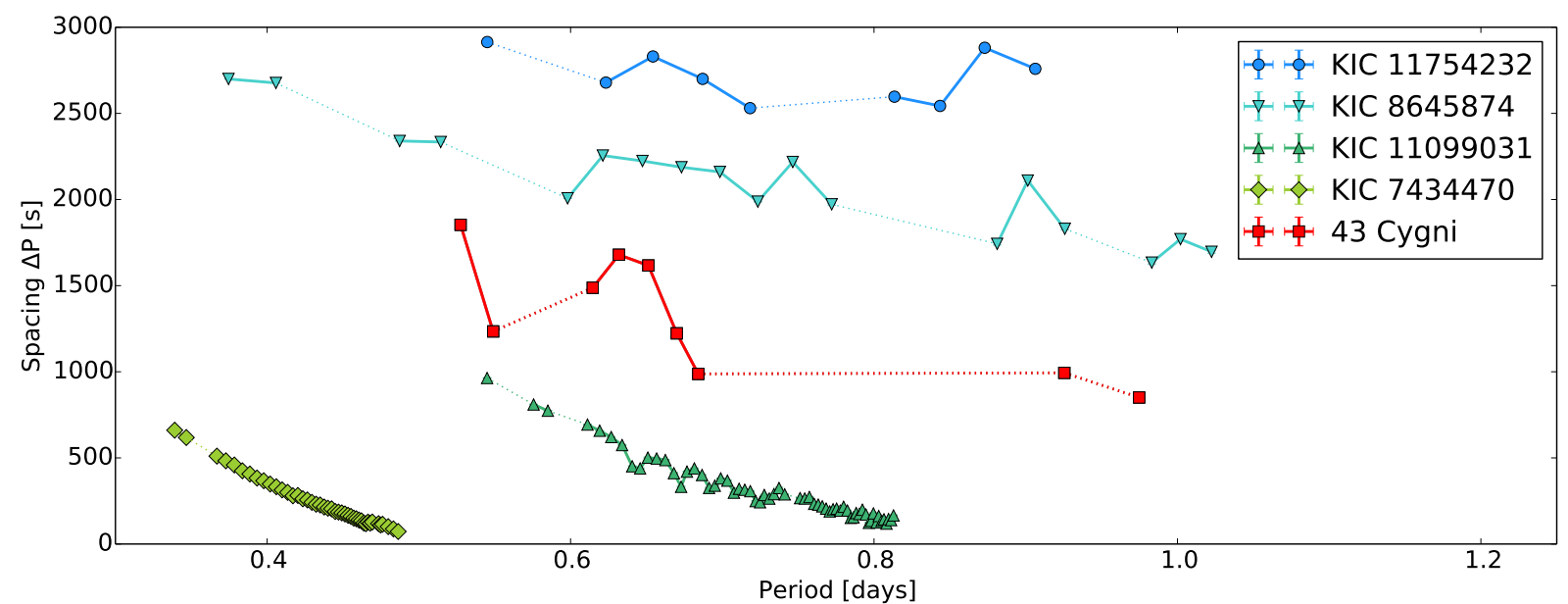

Fig. 6. Comparison of the period-spacing pattern of $43 \mathrm{Cyg}$ with a selection of period-spacing patterns detected for $\gamma$ Dor stars observed with the Kepler space mission. The non-uniform variations of the period-spacings of $43 \mathrm{Cyg}$, caused by chemical gradients in the stellar interior, are clearly visible. The tilt in the pattern, caused by the stellar rotation, follows the trend established by the other observations.
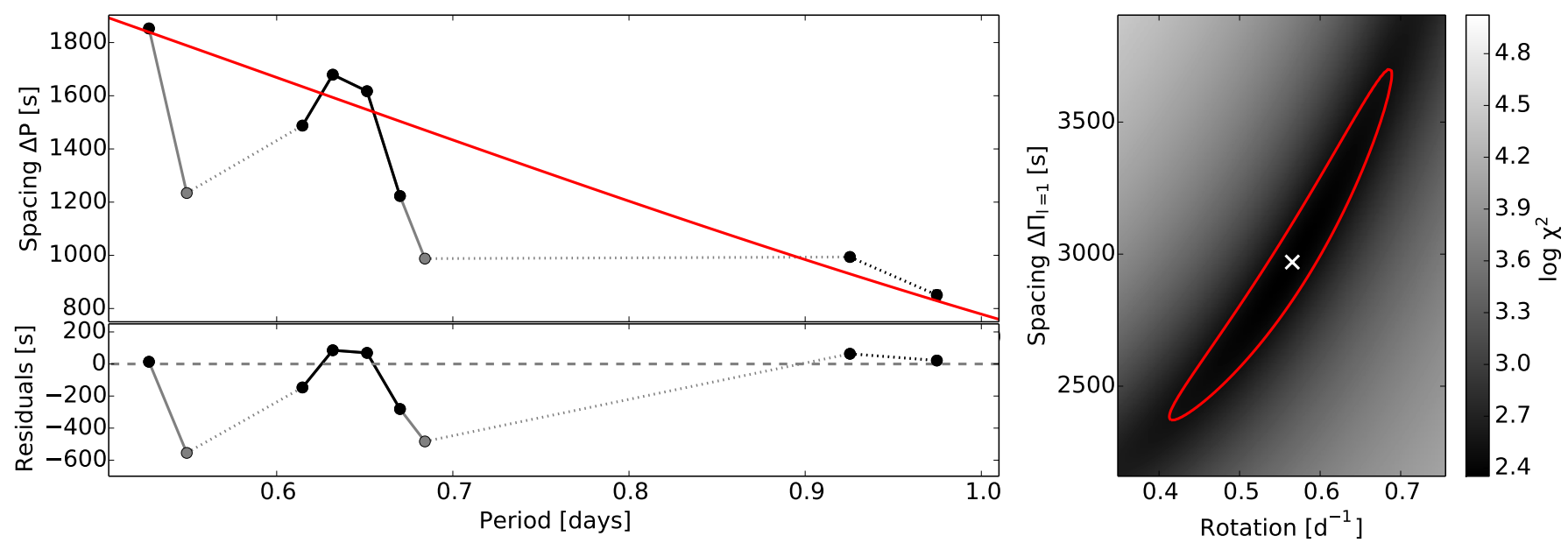

Fig. 7. Top left: observed period spacing pattern (black) with the best asymptotic model pattern (full red line). The largest dips in the pattern (grey) were not taken into account for the model fit. Bottom left: residuals of the model fit to the observed period spacings. Right: $\chi^{2}$-landscape of the fit, with the location of the best model (white cross) and the $1 \sigma$ confidence region (red contour). 


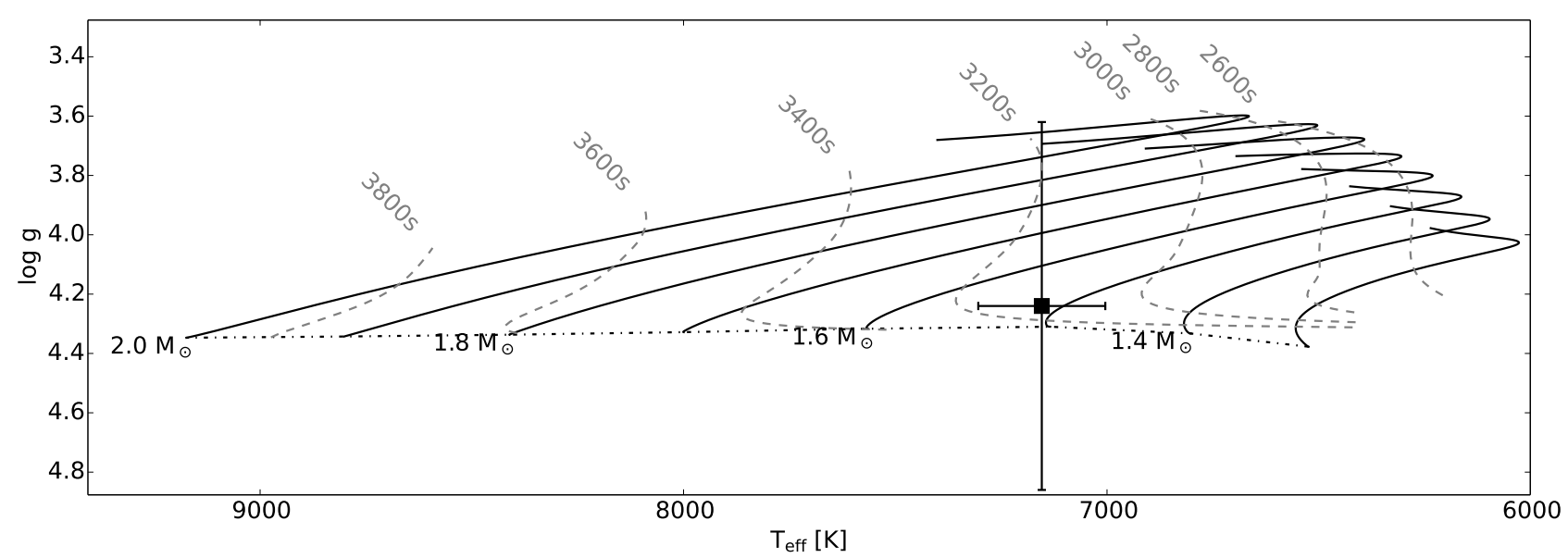

Fig. 8. Location of $43 \mathrm{Cyg}$ (black square) in a Kiel diagram. The evolution tracks (full black lines) have been computed with MESA v7385, assuming solar metallicity and exponential overshooting $f_{o v}=0.015$. The zero-age main sequence (black dash-dotted line) and the values of the asymptotic spacing $\Delta \Pi_{l=1}$ (grey dashed lines) are also shown.

\section{Summary and conclusions}

We have identified a period-spacing pattern for the $\gamma$ Doradus star 43 Cyg from the 156-day long photometric time series obtained by the BRITE-Toronto nano-satellite. This pattern can be used to constrain the star's near-core rotation. We also used high-resolution spectroscopic data to redetermine the star's fundamental parameters to be $T_{\text {eff }}=7150 \pm 150 \mathrm{~K}, \log g=4.2 \pm$ $0.6, v \sin i=44 \pm 4 \mathrm{~km} \mathrm{~s}^{-1}$, and $v_{\text {micro }}=2.99 \pm 0.37 \mathrm{~km} \mathrm{~s}^{-1}$. The atmosphere of $43 \mathrm{Cyg}$ shows a solar chemical composition with no peculiarities.

Of the 43 identified pulsation frequencies in $43 \mathrm{Cyg}, 18$ are part of the identified period-spacing pattern, while 19 frequencies could be explained as combination frequencies. The remaining pulsation frequencies are possible isolated pulsations with different mode properties than those of the detected pattern or are part of a different, yet unidentified spacing pattern.

The detected period-spacing pattern of 43 Cyg clearly shows a downward slope and strong non-uniform variations that indicate a chemical gradient in the stellar interior. We identified the 18 modes of the pattern to be prograde dipole modes and used them to determine the near-core rotation rate of $43 \mathrm{Cyg}$ as $f_{\text {rot }}=0.56_{-0.14}^{+0.12} \mathrm{~d}^{-1}$ and the asymptotic period spacing $\Delta \Pi_{l=1}$ as $2970_{-570}^{+700} \mathrm{~s}$. The relative amplitude of the dips in the observed period-spacing pattern is indicative of one or more strong chemical gradients in the interior of $43 \mathrm{Cyg}$. Theoretical patterns computed with MESA/GYRE for the cases without extra diffusive mixing and with a low amount of mixing $D_{\text {mix }}$ of $1 \mathrm{~cm}^{2} \mathrm{~s}^{-1}$, which reduces the steepness of the chemical gradients in the models, indeed show a significant difference in the shape of the dips, which range from very pronounced to strongly washed out.

The limits of our analysis are determined by the relatively short detected period-spacing pattern that also shows gaps. A detailed asteroseismic modelling can only be conducted based on a longer pattern derived from a significantly longer time base of observations.

Acknowledgements. K.Z. acknowledges support by the Austrian Fonds zur Förderung der wissenschaftlichen Forschung (FWF, project V431-NBL). T.V.R. gratefully acknowledges support from the Fund for Scientific Research of Flanders (FWO), Belgium, under grant agreement G.0B69.13. S.G. was supported by the University of Innsbruck through the "Nachwuchsförderung 2015" project entitled "Asteroseismology with BRITE-Constellation" (PI: K. Zwintz). The research leading to these results has (partially) received funding from the European Research Council (ERC) under the European Union's Horizon 2020 research and innovation programme (grant agreement No. 670519: MAMSIE).
T.V.R. is grateful to the Kavli Institute of Theoretical Physics at the University of California, Santa Barbara, for the kind hospitality during the scientific research visit during which part of the present research was conducted, and which was supported by the Fund for Scientific Research of Flanders (FWO), Belgium (grant agreement V427217N) and in part by the National Science Foundation under grant No. NSF PHY11-25915. We are grateful to Bill Paxton and Richard Townsend, and their collaborators at the Universities of California at Santa Barbara and Wisconsin-Madison, for their valuable work on the stellar evolution code MESA and stellar pulsation code GYRE. A. Pigulski and A. Popowicz acknowledge support from the Polish National Science Centre (grant No. 2016/21/B/ST9/01126 and 2016/21/D/ST9/00656). The Polish contribution to the BRITE mission is funded by the Polish National Science Centre (NCN, grant 2011/01/M/ST9/05914) and a SPUB grant by the Polish Ministry of Science and Higher Education. G.H. acknowledges support by the Polish NCN grant 2015/18/A/ST9/00578. G.A.W. acknowledges Discovery Grant support from the Natural Sciences and Engineering Research Council (NSERC) of Canada. A.F.J.M. is grateful for financial aid from NSERC (Canada) and FQRNT (Quebec).

\section{References}

Asplund, M., Grevesse, N., Sauval, A. J., \& Scott, P. 2009, ARA\&A, 47, 481 Balona, L. A. 2014, MNRAS, 439, 3453

Baran, A. S., Koen, C., \& Pokrzywka, B. 2015, MNRAS, 448, L16

Bedding, T. R., Murphy, S. J., Colman, I. L., \& Kurtz, D. W. 2015, in European Physical Journal Web of Conferences, 101, 01005

Bouabid, M.-P., Dupret, M.-A., Salmon, S., et al. 2013, MNRAS, 429, 2500

Cantiello, M., Mankovich, C., Bildsten, L., Christensen-Dalsgaard, J., \& Paxton, B. 2014, ApJ, 788, 93

Chapellier, E., Mathias, P., Weiss, W. W., Le Contel, D., \& Debosscher, J. 2012, A\&A, 540, A117

Cuypers, J., Aerts, C., De Cat, P., et al. 2009, A\&A, 499, 967

David, T. J., \& Hillenbrand, L. A. 2015, ApJ, 804, 146

Dupret, M.-A., Grigahcène, A., Garrido, R., Gabriel, M., \& Scuflaire, R. 2005, A\&A, 435, 927

Eckart, G. 1960, Hydrodynamics of Oceans and Atmospheres (Oxford: Pergamon Press)

Fekel, F. C., Warner, P. B., \& Kaye, A. B. 2003, AJ, 125, 2196

Gerbaldi, M., Faraggiana, R., \& Caffau, E. 2007, A\&A, 472, 241

Guo, Z., Gies, D. R., \& Matson, R. A. 2017, ApJ, submitted [arXiv: 1704.03789]

Guzik, J. A., Kaye, A. B., Bradley, P. A., Cox, A. N., \& Neuforge, C. 2000, ApJ, 542, L57

Handler, G. 1999, MNRAS, 309, L19

Henry, G. W., Fekel, F. C., \& Henry, S. M. 2005, AJ, 129, 2815

Jankov, S., Mathias, P., Chapellier, E., Le Contel, J.-M., \& Sareyan, J.-P. 2006, A\&A, 453, 1041

Kaye, A. B., Handler, G., Krisciunas, K., Poretti, E., \& Zerbi, F. M. 1999, PASP, 111,840

Keen, M. A., Bedding, T. R., Murphy, S. J., et al. 2015, MNRAS, 454, 1792

Koch, D. G., Borucki, W. J., Basri, G., et al. 2010, ApJ, 713, L79 
Kochukhov, O. P. 2007, in Physics of Magnetic Stars, eds. I. I. Romanyuk, D. O Kudryavtsev, O. M. Neizvestnaya, \& V. M. Shapoval, 109

Kupka, F., Piskunov, N., Ryabchikova, T. A., Stempels, H. C., \& Weiss, W. W. 1999, A\&AS, 138, 119

Kurtz, D. W., Saio, H., Takata, M., et al. 2014, MNRAS, 444, 102

Lee, U., \& Saio, H. 1987, MNRAS, 224, 513

Lee, U., \& Saio, H. 1997, ApJ, 491, 839

Marques, J. P., Goupil, M. J., Lebreton, Y., et al. 2013, A\&A, 549, A74

Miglio, A., Montalbán, J., Noels, A., \& Eggenberger, P. 2008, MNRAS, 386, 1487

Moravveji, E., Aerts, C., Pápics, P. I., Triana, S. A., \& Vandoren, B. 2015, A\&A, 580, A27

Moravveji, E., Townsend, R. H. D., Aerts, C., \& Mathis, S. 2016, ApJ, 823, 130

Murphy, S. J., Fossati, L., Bedding, T. R., et al. 2016, MNRAS, 459, 1201

Ouazzani, R.-M., Salmon, S. J. A. J., Antoci, V., et al. 2017, MNRAS, 465, 2294

Pablo, H., Whittaker, G. N., Popowicz, A., et al. 2016, PASP, 128, 125001

Paxton, B., Bildsten, L., Dotter, A., et al. 2011, ApJS, 192, 3

Paxton, B., Cantiello, M., Arras, P., et al. 2013, ApJS, 208, 4

Paxton, B., Marchant, P., Schwab, J., et al. 2015, ApJS, 220, 15

Perryman, M. A. C., Lindegren, L., Kovalevsky, J., et al. 1997, A\&A, 323, L49

Pigulski, A., Cugier, H., Popowicz, A., et al. 2016, A\&A, 588, A55

Piskunov, N., \& Valenti, J. A. 2017, A\&A, 597, A16

Piskunov, N., Ryabchikova, T., Yu. Pakhomov, Y., et al. 2017, in Stars: From Collapse to Collapse, eds. Y. Y. Balega, D. O. Kudryavtsev, I. I. Romanyuk, \& I. A. Yakunin, ASP Conf. Ser., 510, 509
Popowicz, A., Pigulski, A., Bernacki, K., et al. 2017, A\&A, 605, A26 Prat, V., Mathis, S., Lignières, F., Ballot, J., \& Culpin, P.-M. 2017, A\&A, 598, A105

Raskin, G., van Winckel, H., Hensberge, H., et al. 2011, A\&A, 526, A69

Rogers, T. M. 2015, ApJ, 815, L30

Rogers, T. M., Lin, D. N. C., McElwaine, J. N., \& Lau, H. H. B. 2013, ApJ, 772, 21

Ryabchikova, T., Piskunov, N., Pakhomov, Y., et al. 2016, MNRAS, 456, 1221

Saio, H., Kurtz, D. W., Takata, M., et al. 2015, MNRAS, 447, 3264

Schmid, V. S., \& Aerts, C. 2016, A\&A, 592, A116

Schwarzenberg-Czerny, A. 2003, in Interplay of Periodic, Cyclic and Stochastic Variability in Selected Areas of the H-R Diagram, ed. C. Sterken, ASP Conf. Ser., 292, 383

Shulyak, D., Tsymbal, V., Ryabchikova, T., Stütz, C., \& Weiss, W. W. 2004, A\&A, 428, 993

Sowicka, P., Handler, G., Dẹbski, B., et al. 2017, MNRAS, 467, 4663

Tassoul, M. 1980, ApJS, 43, 469

Tkachenko, A., Lehmann, H., Smalley, B., Debosscher, J., \& Aerts, C. 2012, MNRAS, 422, 2960

Townsend, R. H. D., \& Teitler, S. A. 2013, MNRAS, 435, 3406

Triana, S. A., Moravveji, E., Pápics, P. I., et al. 2015, ApJ, 810, 16

Valenti, J. A., \& Piskunov, N. 1996, A\&AS, 118, 595

Van Reeth, T., Tkachenko, A., Aerts, C., et al. 2015a, A\&A, 574, A17

Van Reeth, T., Tkachenko, A., Aerts, C., et al. 2015b, ApJS, 218, 27

Van Reeth, T., Tkachenko, A., \& Aerts, C. 2016, A\&A, 593, A120

Weiss, W. W., Rucinski, S. M., Moffat, A. F. J., et al. 2014, PASP, 126, 573 